\title{
Middle Cerebral Artery Occlusion Model in Rodents: Methods and Potential Pitfalls
}

\author{
Fudong Liu' ${ }^{1}$ and Louise D. McCullough ${ }^{1,2}$ \\ ${ }^{1}$ Department of Neuroscience, University of Connecticut Health Center, Farmington, CT 06030, USA \\ ${ }^{2}$ Department of Neurology, University of Connecticut Health Center and The Stroke Center at Hartford Hospital, \\ 263 Farmington Avenue, Farmington, CT 06030, USA \\ Correspondence should be addressed to Louise D. McCullough, lmccullough@uchc.edu
}

Received 15 September 2010; Revised 23 December 2010; Accepted 23 December 2010

Academic Editor: Andrea Vecchione

Copyright (๑) 2011 F. Liu and L. D. McCullough. This is an open access article distributed under the Creative Commons Attribution License, which permits unrestricted use, distribution, and reproduction in any medium, provided the original work is properly cited.

\begin{abstract}
A variety of animal models have been developed for modeling ischemic stroke. The middle cerebral artery occlusion (MCAO) model has been utilized extensively, especially in rodents. While the MCAO model provides stroke researchers with an excellent platform to investigate the disease, controversial or even paradoxical results are occasionally seen in the literature utilizing this model. Various factors exert important effects on the outcome in this stroke model, including the age and sex of the animal examined. This paper discusses emerging information on the effects of age and sex on ischemic outcomes after MCAO, with an emphasis on mouse models of stroke.
\end{abstract}

\section{Introduction}

Stroke is the third major cause of mortality and the leading cause of long-term disability in the United States. Ischemic stroke accounts for approximately $80 \%$ of all strokes [1]; however, only one FDA- approved therapy exists for treatment of acute ischemic stroke, the thrombolytic tissue plasminogen activator (tPA) [2]. In order to investigate the mechanisms underlying injury after ischemic stroke as well as to develop effective therapeutic approaches to the disease, several ischemic stroke models have been developed in a variety of species, including rodents, canines, rabbits, cats, and even nonhuman primates [3-6]. Models of stroke that can be used in rodents are becoming increasingly popular at bench because (1) genetically-engineered mice are particularly useful for furthering our understanding of components of ischemic pathophysiology and in designing potential new preventative, neuroprotective, and therapeutic drugs and interventions; (2) a number of neurosensory and motor behavior outcomes have become standardized for rodents, which facilitates the assessment of functional outcomes after experimental stroke [7]; (3) the public tends to have fewer animal welfare concerns regarding rodents in research compared to nonrodents [7-10]. In general, there are four major types of animal models of ischemic stroke: (1) complete global cerebral ischemia; (2) incomplete global ischemia; (3) focal cerebral ischemia and (4) Multifocal cerebral ischemia [10] (Figure 1). Since ischemic stroke in patients usually results from a thrombotic or embolic occlusion in a major cerebral artery, most often the middle cerebral artery (MCA), experimental focal cerebral ischemia models have been developed to mimic human stroke and serve as an indispensable tool in the stroke research field [11].

However, all experimental models of disease have weaknesses and stroke modeling is no exception, especially with the reliance on rodent models. Despite many promising pharmacological agents that dramatically reduce tissue injury after experimental stroke at the bench, none have been translated to efficacious agents for clinical use. Possible reasons for this "lack of translation" have been recently reviewed elsewhere $[12,13]$, but some of the most obvious but frequently ignored factors will be discussed here, that is, the effect of age and sex on stroke outcomes. Most experimental stroke studies have been done exclusively in young male animals, although stroke is a disease that mainly affects the elderly [14]. Stroke is also sexually dimorphic 

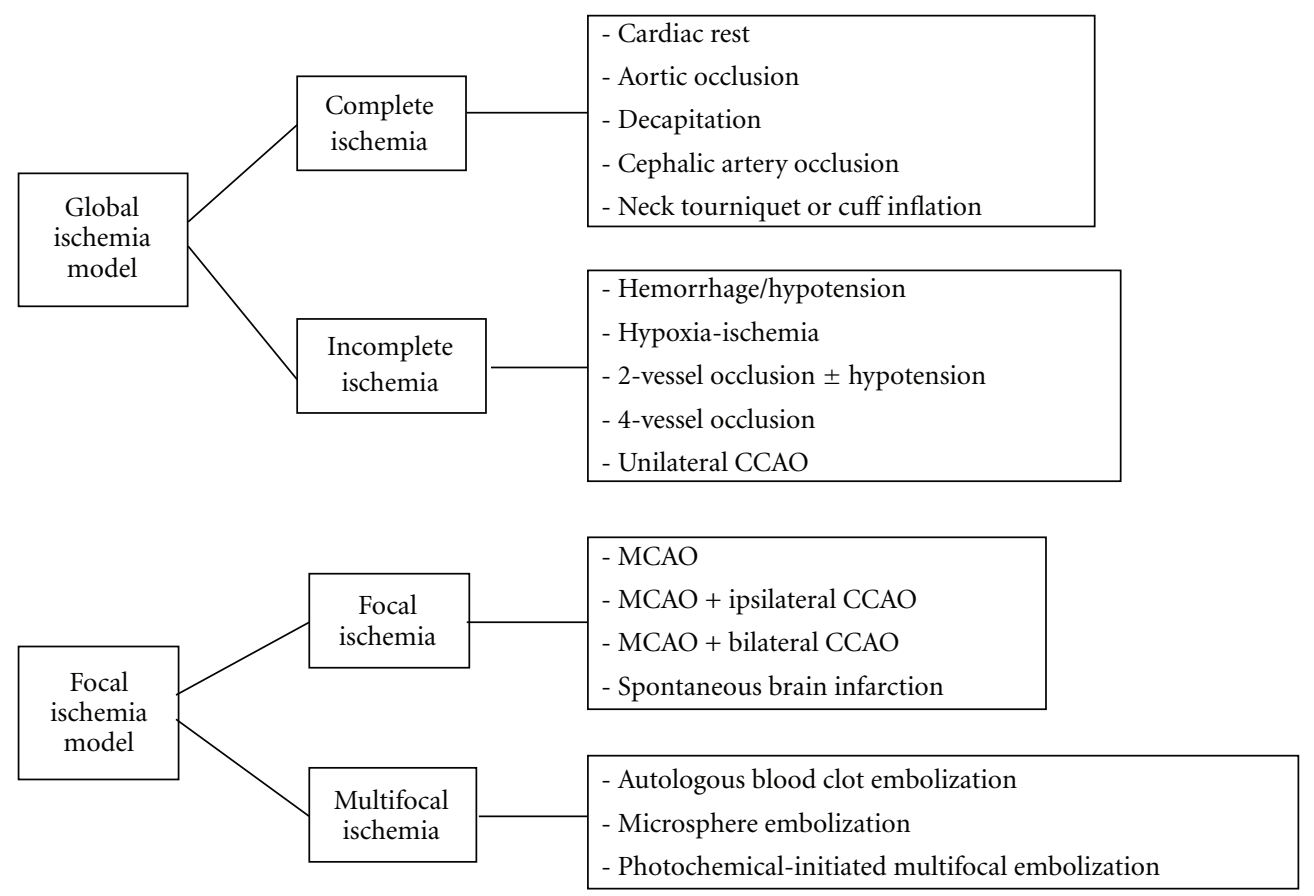

FIGURE 1: Major types of animal models of ischemic stroke.

in clinical populations with different functional outcomes, age of onset, and etiologies in men and women [15]. We will describe the most commonly used models in the investigation of stroke in rodents, with a focus on the MCAO model, and discuss some of the limitations of this model as it is currently used. Several common factors that can influence histological and behavioral outcomes including aging and sex/hormonal factors will also be discussed.

\section{Modeling Stroke: MCAO Model}

Four major rodent models of focal cerebral ischemia have been developed: (1) models not requiring craniotomy (embolic model, intraluminal suture MCAO model, photothrombosis model, and endothelin-1-induced stroke model), (2) models requiring craniotomy (Tamura's model), (3) posterior cerebral circulation stroke models, and (4) cerebral venous thrombosis models. These have been the subject of an excellent comprehensive review [11] and will not be discussed here except for the intraluminal model. Among these well-developed models, MCAO is widely used and has been extended to the mouse in recent genetic studies of cell death mechanisms. This model offers a simpler and less traumatic surgical approach compared with craniotomy models, lends itself more readily to the study of reperfusion and has been adapted for use in continuous magnetic resonance imaging [16], produces focal occlusion of a large cerebral artery as seen in human stroke, and can be done in a high-throughput manner [17]. Rigorous control of temperature, physiological variables, and assessment of occlusion with noninvasive means (i.e., Laser Doppler) reduces variability.
2.1. Infarct Size Induced by MCAO. The reversible MCAO model has been well studied and utilized by stroke researchers due to its clinically relevant nature that mimics recanalization of an embolized or thrombosed vessel by tPA (Figure 2). Although the model has been employed to study ischemic stroke for decades, the evolution of infarct within the area blood supplied by MCA has not been well elucidated. Therefore significant controversy exists due to the variability of final infarct size and debate as to the most reliable time point to measure the effects of various therapeutic agents $[9,18,19]$. Generally speaking, infarct development appears to be more rapid in mice than in rats as well as in reperfusion versus permanent "nonreperfusion" MCAO models. Previous studies have demonstrated an increase in infarct volumes even as late as $3 \mathrm{~d}$ after stroke in rats $[20,21]$; however these animals had no restoration of blood flow which may delay infarct development. In a previous study [16] we have shown that 90-minute occlusion of the MCA induces a peak volume of injury as delineated by TTC staining by $24 \mathrm{~h}$ in mice which remains unchanged through day 7 of reperfusion (Figure 3). We also observed a spatiotemporal evolution of core and penumbra: at earlier time points ( 2 and 6 hours after occlusion), the histological infarct core, as measured by TTC, is limited to the striatum, and the viable tissue surrounded this core and included much of the cortex. Subsequently the "TTC-defined" core expands to involve most of the cortical tissue supplied by the MCA. This occurs quite rapidly and is complete (by TTC) within 6-12 hours after stroke onset. It is unclear if any intervention begun several hours after ischemic onset can reduce recruitment of the penumbra. Certainly by 12 hours, all of the territory supplied by the MCA is irreversibly damaged. Studies with MRI in rat models of transient 


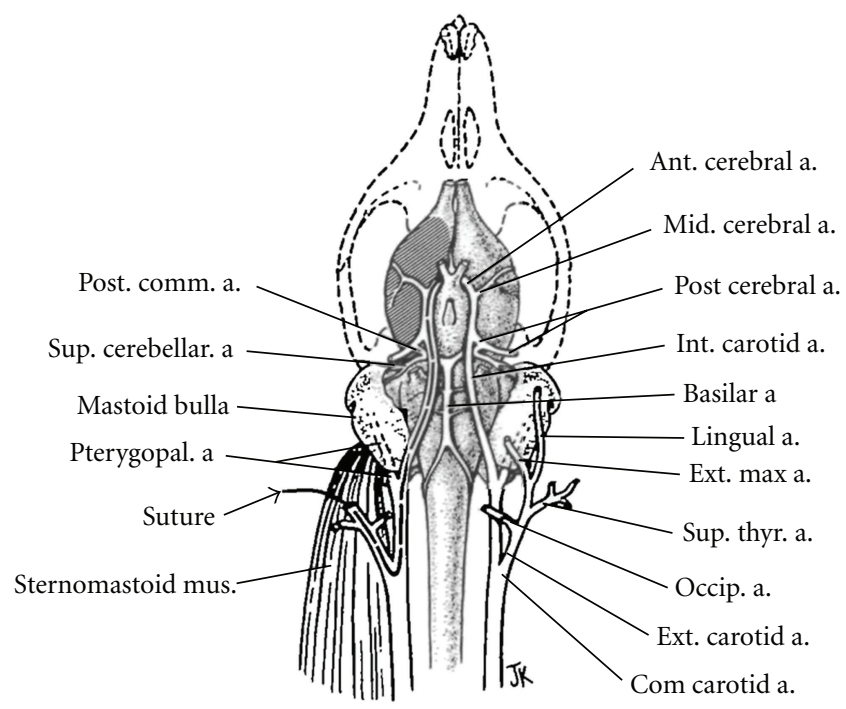

FIGURE 2: Diagram of cerebrovascular anatomy and MCAO in rats. Reprinted with permission from Longa, EZ et al., Stroke 1989 20: 84-91. a., artery; Com.: common; Ext.: external; Int.: internal.

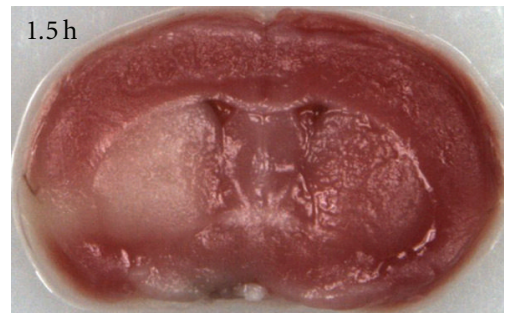

(a)

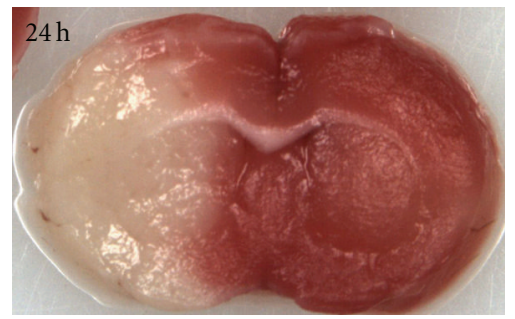

(d)

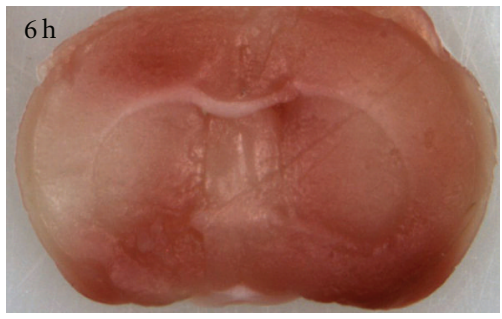

(b)

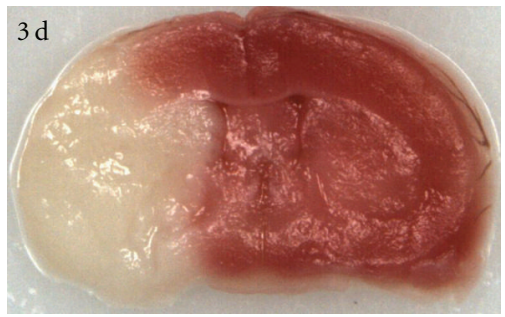

(e)

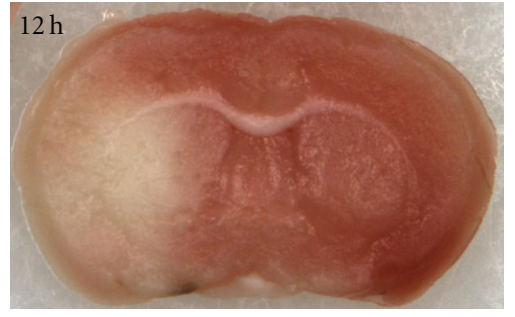

(c)

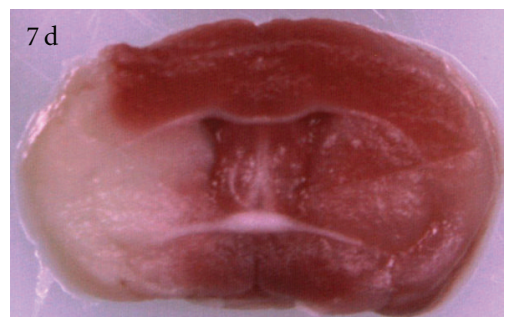

(f)

FIGURE 3: Evolution of infarct in mouse brain after 90-minute MCAO.

MCAO [22, 23] demonstrated that stroke-induced infarct evolved into an area of injury of similar magnitude to the initial diffusion disturbance by $24 \mathrm{~h}$ as measured by a reduced apparent diffusion coefficient and prolonged T2 signals. Several previous studies have also revealed that apoptosis induced by transient MCAO model reached its peak at $24-48 \mathrm{~h}$ of stroke $[24,25]$. In murine reperfusion models, increases in T2 signal were seen extremely early (by 90 minutes) in the infarct area, indicative of edema and breakdown of the blood-brain barrier, which were not seen after permanent occlusion. This suggests that reperfusion may accelerate infarct development, at least in mice [26]. The dynamics of the penumbra are also different between strains, as measured by MRI [27]. The spontaneously hypertensive rat and its stroke-prone cohort develop larger and much less variable infarcts following MCAO [28]. The rapid evolution of the penumbra does emphasize one of the limitations in clinical neuroprotection studies. Most animal studies have administered a putative neuroprotective agent either pre-ischemia, intraischemia, or very soon after reperfusion [29-31], time windows that are impossible to mimic in clinical trials. Although a prolonged "therapeutic window" does allow for a greater number of patients to be treated, it is unlikely that salvageable tissue is present, especially after successful reperfusion if it occurs after 8 hours. Attempting to treat patients without radiographic evidence of penumbral tissue after 6 hours is likely an exercise in futility and dilutes any potential benefit of an acute 
neuroprotective agent. Other mechanisms that predominate after injury, such as inflammation, may be more viable targets [32].

Interestingly, different histological staining methods reveal different evolving patterns of infarct tissue or degenerating neurons. Each method has advantages and disadvantages. For example, while TTC staining of MCAO-induced brain injury demonstrated no further enlargement of infarct after $24 \mathrm{~h}$ of stroke, Fluoro-Jade B [33] staining showed neuronal degeneration peaked at $24 \mathrm{~h}$, increased by 48 hours but decreased to baseline values by $7 \mathrm{~d}$ after stroke [16]. This suggests that Fluoro-Jade B is a more sensitive marker for acute neuronal degeneration but may not be as useful for assessment of chronic damage, which is more accurately performed with cresyl violet $(\mathrm{CV})$. TTC is a marker of tissue dehydrogenase and mitochondrial dysfunction and may not represent irreversible cell death, therefore it may overestimate infarct size [34]. Longer-term assessment of infarct also suggests that damage, as measured by acute TTC staining, overestimates the degree of injury. 50\% of the ischemic hemisphere is poorly stained by TTC acutely, but at 30 days, only $10-20 \%$ of the hemispheric volume is lost (unpublished observations). This suggests that TTC may not reliably reflect tissue death, but rather tissue dysfunction. Fluoro-Jade B is very specific for neuronal death but becomes inaccurate after several days, as the degenerating neurons have often been removed, leading to an underestimate of neuronal injury. CV is good for long-term studies but is timeconsuming. A combination of methods is likely the most accurate, and both short-(24-72 hrs) and long-term (weeks) histological outcomes should be assessed prior to clinical development of any neuroprotective agent.

2.2. Modeling Stroke Risk Factors. Increased stroke risk factors are major contributing factors to the rising prevalence of stroke and include hypertension, obesity, and diabetes [35]. Models for these important risk factors have been developed. For example, the APO E-deficient (apoE-/-) mouse, established in 1992, is of the most important animal models of atherosclerosis [36]. Spontaneously hypertensive $(\mathrm{SH})$ rats are utilized as a "stroke-prone" animal model and widely used in hypertension studies [37]. Mutation of cystathionine $\beta$-synthetase (CBS), which induces enhanced atherothrombosis [38], has also been investigated. Although modeling stroke risk factors has advanced our knowledge of these disorders, the interaction with age and sex has been frequently ignored.

\section{Effect of Age on Modeling MCAO}

Aging is the most important independent risk factor for stroke. Older patients have higher in-hospital mortality as well as poorer functional outcomes after an ischemic event [14]. Although age is one of the most significant prognostic markers for poor outcome [39], very few studies have been performed in aged animals, especially in animals over 15 months of age. Numerous neurochemical and physiological changes occur with aging [40]. Aging animals have less edema formation after stroke compared to the young [41], and NKCC, a Na-K-Cl cotransporter, is expressed at a lower level in aging mice than in young mice after MCAO [42], suggesting a possible mechanism for this finding. Clinical postmortem studies [43] also confirmed more robust edema formation in the young brain after stroke and is in part the rationale for proposing an upper age limit of 60 for hemicraniectomy [44]. Recent data suggests that ischemic preconditioning is less effective in the aged heart in both experimental and clinical studies [45], and our study also found that Compound $\mathrm{C}$, adenosine monophosphate-activated protein kinase (AMPK) inhibitor which has been proved to be neuroprotective in young animals after MCAO [46, 47], has no effect in aging animals (manuscript submitted). The continued clinical failure of promising neuroprotective agents [48] has led us to question the appropriateness of modeling stroke in young animals. New paradigms for conducting translational research in aging animals are urgently needed.

It is far more difficult to perform MCAO in aging rodents. Aging animals are usually heavier with higher amounts of visceral fat, are less tolerant to anesthesia, and have less flexible vessels making insertion of occluding sutures much more difficult than in young animals. Aging animals have higher mortality after MCAO due to frailty, peripheral immunesupression, and other comorbid diseases [41]. Stroke researchers frequently avoid using aging animals for MCAO due to the more complex surgical procedure and high cost of purchasing and raising animals. Very few experimental studies exist in the literature that have examined middle aged and aging animals, and these have led to somewhat inconsistent results. Kharlamov et al. [49] found no difference in infarct size at 24 hours when male Fisher rats of three different age groups (4, 20, and 27 months old) were evaluated. In another study, age was associated with an increase in infarct size from 9 to $12 \%$ in the neocortex and striatum in aged male Wistar rats [50]. In contrast, other studies have suggested that histological infarct damage may be paradoxically higher in young ( 3 months) compared to old (24-26 months) male rats [51]. Many of the studies that have shown an age-related increase in stroke volume used female animals (as they are smaller than agematched males), and findings may also be related to the loss of ovarian hormones with aging [52]. Our previous studies have definitively shown that aging male mice have significantly smaller infarcts than young males after $24 \mathrm{~h}$ of 90-minute MCAO [41], at least at the histological level. This difference can be detected even as long as $30 \mathrm{~d}$ after MCAO (unpublished data). In spite of the disagreements between the histological effects of aging on infarct volume (with some showing larger and others showing smaller infarct injury), invariably significantly higher mortality rates and more severe neurological impairments are found in the older animals. For example, one study that examined 3-4 month versus 22-24-month-old male rats demonstrated a mortality rate of $43.5 \%$ in aged rats compared to $9 \%$ in the young [53]. Despite the fact that the mortality risk from the ischemic damage is greater for old mice, we have found that eventual recovery ( 4 weeks after stroke) is not different from that of 
a young mouse in most simple behavioral tests, although the "slope" of recovery is much slower. An exception is the corner test (manuscript submitted). The correlation between brain damage, behavioral recovery, and age needs to be further investigated.

Given that young and aging animals are usually of different weights at the time of MCAO, it is not surprising that the cerebral vessels are also of different size and dimension. To ensure adequate and equivalent levels of cerebral blood flow (CBF) reduction, we have utilized different sizes of occluding sutures in young and aging mice. We have found that in $[41,42]$ young mice $(20 \sim 25 \mathrm{mg})$, successful occlusion requires a $0.21 \mathrm{~mm}$ suture, but in aging mice $(30 \sim 40 \mathrm{mg})$, a larger $0.23 \mathrm{~mm}$ suture is required to obtain the same degree of $\mathrm{CBF}$ reduction. Our experience emphasizes the importance of routine laser Doppler blood flow monitoring to ensure adequate ( $85 \%$ drop from prestroke baseline) in murine MCAO models. This not only reduces variability but also ensures that each animal experiences the same level of cerebral ischemia, as this can have dramatic effects on outcomes. Several other issues need to be considered when utilizing aging animals in research, including the higher rates of infections and malignancy.

\section{Effect of Sex on Modeling MCAO}

It is increasingly recognized that the epidemiology of ischemic stroke is sexually dimorphic [15], and therapeutic agents aimed at stroke function differently in male and female subjects. For example, anticoagulated women had a relative risk of 2.0 for ischemic stroke versus anticoagulated men [54], suggesting sex differences in coagulation. Women experience a greater benefit from tissue plasminogen activator (tPA) treatment than men [55]. Clinical trials of the 21-aminosteroid lipid peroxidation inhibitor tirilazad conducted in traumatic brain injury, subarachnoid hemorrhage, ischemic stroke, and spinal cord injury indicated that the beneficial effect of tirilazad was greater in males than females $[56,57]$. These sex differences are becoming increasingly apparent in clinical populations. The vast majority of preclinical studies also continue to exclusively use male animals. There are significant limitations to this approach, as we discuss here, utilizing estrogen $\left(\mathrm{E}_{2}\right)$ as an example. Prior to menopause, women have a lower risk of stroke relative to age-matched men [58]. After menopause, the incidence of stroke in women increases [59], coincident with the diminished circulating levels of estrogen and progesterone and surpasses that of men. Therefore, this pattern of stroke risk has been attributed to the presence of protective female sex hormones. Over the past thirty years, the majority of cohort, retrospective, or prospective observational studies have demonstrated significant reductions in cardiovascular disease in postmenopausal women receiving $\mathrm{E}_{2}$ (ERT) or combined estrogen-progestin therapy (HRT) [60]. A growing number of preclinical studies have also documented sexual dimorphism in stroke. Female rats and mice of many different inbred and outbred strains sustain smaller tissue damage for an equivalent insult from focal or global cerebral ischemia [61]. The "female-protected" phenotype can often be reversed by inducing surgical menopause via ovariectomy, and protection can be recapitulated in male animals by administration of exogenous estrogens [62]. $E_{2}$ has neurotrophic, antiapoptotic, vasodilatory, anti-inflammatory, and antioxidant effects, each of which could contribute to improved outcome in the brains of males and females [63]. $\mathrm{E}_{2}$ treatment at physiological relevant concentrations reduces infarction after MCAO in ovariectomized (OVX) or reproductively senescent female animals $[64,65]$, as well as in males [61, 66], even when given after MCAO [62].

The Woman's Health Initiative (WHI), the largest clinical trial of $\mathrm{E}_{2}$ replacement for stroke prevention, showed a surprising increase in stroke incidence in estrogen-treated woman $[67,68]$. The explanation for these findings has been debated extensively in the literature [67] but is thought to be due to the unexpected proinflammatory effects of estrogen administration after a long period of "hypoestrogenemia". A recent study in mice demonstrated that prolonged loss of $\mathrm{E}_{2}$ prior to replacement (10 weeks) ameliorated the neuroprotective and anti-inflammatory effects of $E_{2}$ seen in MCAO-model [69]. It appears that timing of estrogen replacement after the menopause is critical to outcome in both animal models and clinical trials. Women involved in the WHI trial were considerably older (average age of 63) and well past menopause (which occurs at an average age of 51) prior to randomization [70, 71]. In the Northern Manhattan Stroke Study, stroke rates in women do not equalize to those of men until beyond 75 years of age suggesting that considerable time passes before females lose the protection of gonadal steroids.

In addition to the timing of $\mathrm{E}_{2}$ replacement, other factors may also affect the effects of $E_{2}$ in MCAO induced brain injury, including dosing, the age of the animal, and the timing of replacement. A recent study [72] administering $17 \beta$-estradiol to OVX rats that underwent MCAO found that slow-release commercially purchased pellets of $17 \beta$-estradiol produced an early supraphysiological peaks followed by a substantial decrease in serum levels of $\mathrm{E}_{2}$, while silastic capsules (inner/outer diameter: 1.575/3.175 mm) yielded $17 \beta$-estradiol concentrations within the physiological range for at least 4 weeks. The former was detrimental and exacerbated brain injury, whereas the latter was beneficial in reducing MCAO-induced brain damage. The stroke model itself may also exert an influence on the effect of $\mathrm{E}_{2}$ treatment. Although most studies with transient MCAO confirmed the neuroprotective effect of $\mathrm{E}_{2}$ replacement [63], 17 $\beta$-estradiol treatment following permanent $\mathrm{MCAO}$ did not improve stroke outcomes; however commercial pellets were used, and supraphysiological levels of $E_{2}$ could account for this discrepancy [73].

Interestingly, it is becoming increasingly accepted that the effect of sex on stroke outcome may also be hormone independent. Innate sex differences also contribute to the variability of infarct volumes induced by MCAO in male and female animals. It is increasingly recognized that different cell death pathways may predominate, respectively, in male and female subjects exposed to ischemic injury [74] (Figure 4). Cytochrome C-caspase pathway was found 


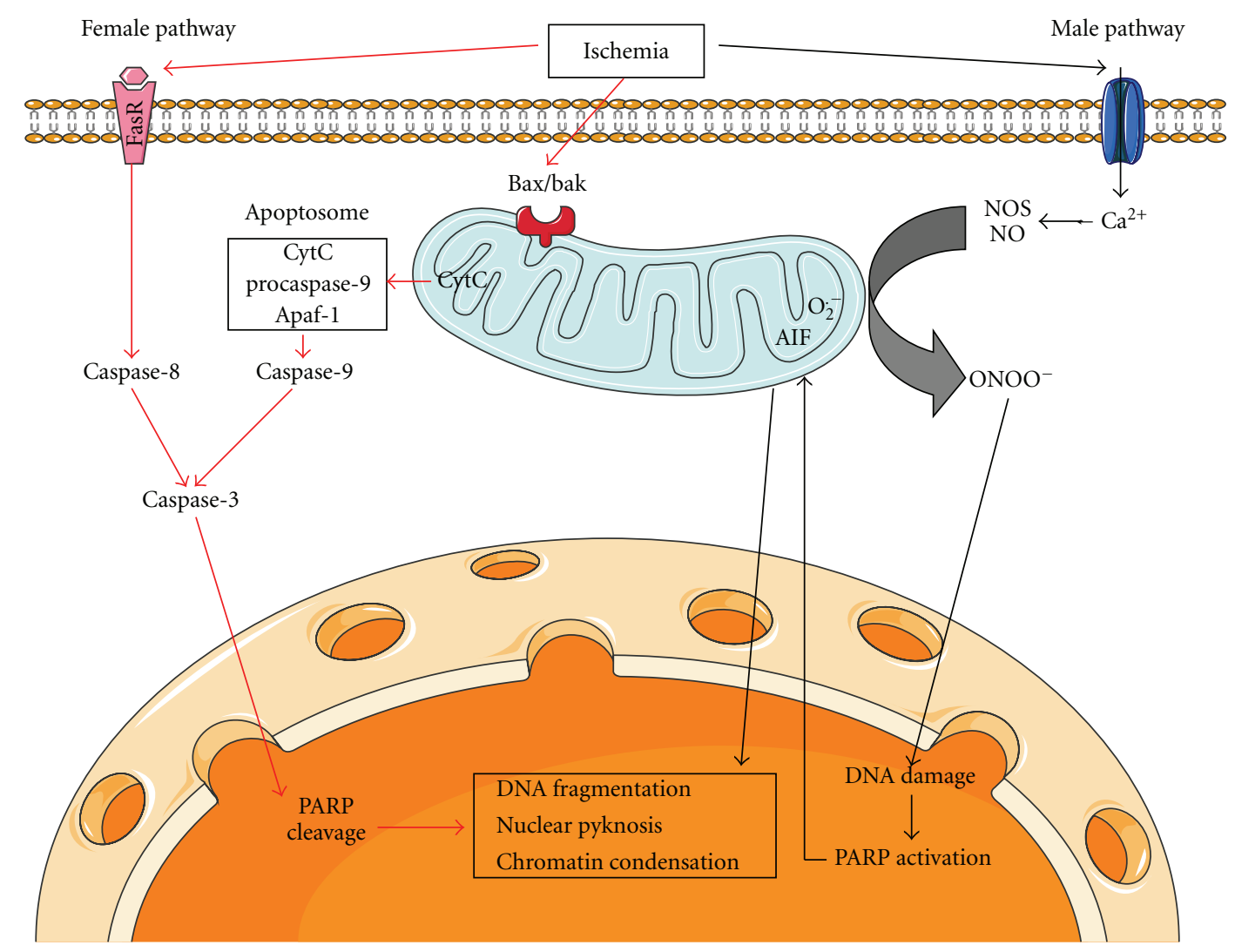

FIGURE 4: Mechanisms of PARP-AIF and cytochrome C-caspase-mediated cell death pathways predominant, respectively, in male and female brains after ischemia. The figure was produced using Servier Medical Art.

to be the predominant cell death pathway in females after ischemic insults [31, 75]. Administration of broad spectrum caspase inhibitor, quinoline-Val-Asp (Ome)-CH2O-phenoxy (Q-VD-OPh), decreased infarct volumes in OVX female mice after MCAO but had no effect in males [31]. On the other hand, PARP-1-AIF pathway mediates cell death induced by ischemic insults mainly in male animals $[76,77]$. The PARP-1 inhibitor minocycline is neuroprotective in male mice but not in OVX females after MCAO [78], which awaits confirmation. Aging female mice exhibited larger infarct volumes than aging males after $24 \mathrm{~h}$ of 90 -minute $\mathrm{MCAO}$, although the serum levels of E2 were equivalently low in both aging males and females [41]. Female rats are more vulnerable to the long-term consequences of neonatal inflammatory injury compared to neonatally injured males [79].

The sexual dimorphism seen in MCAO in aging animals may also be due to differing inflammatory responses to stroke in aging males and females. A growing body of evidence suggests that sexual dimorphism exists in the inflammatory response and even in the frequency of pro/anti-inflammatory gene variants [80]. Leukocyte function varies by sex in aging rats; aging males are less immune competent than age-matched females as females showed higher lymphoproliferative responses, higher Natural Killer activity, and higher IL-2 release in the spleen and axillary nodes [81]. In previous studies in aging mice, [41] we found that the serum levels of IL-6, a potent proinflammatory cytokine [82, 83], were lower in aging males than in aging females after MCAO. Indeed, these sex differences can also be recapitulated in neonatal animals [75], where circulating hormone levels are equivalent [84]. All these studies suggested that sex is an independent viable that must be addressed in translational stroke studies if we hope to develop efficacious neuroprotective agents from our animal models. Sex differences in stroke must be considered as an independent viable when interpreting results from either experimental or clinical trials for neuroprotective agents.

In summary, the MCAO model in rodents provides a useful tool to stroke researchers; nevertheless, timing of analysis, animal age, and sex are important factors that have major effects on stroke outcome but continue to be less studied. We must keep in mind these influential factors when designing our experiments and in our attempts to translate therapies into the clinical populations at the highest risk for stroke, women and the elderly.

\section{Acknowledgment}

This work was supported by the NIH/NINDS (Grants NS050505 and NS055215 to L. D. McCullough). 


\section{References}

[1] W. Rosamond, K. Flegal, K. Furie et al., "Heart disease and stroke statistics-2008 update: a report from the American heart association statistics committee and stroke statistics subcommittee," Circulation, vol. 117, no. 4, pp. e25-e46, 2008.

[2] W. M. Armstead, K. Ganguly, J. W. Kiessling et al., "Signaling, delivery and age as emerging issues in the benefit/risk ratio outcome of tPA for treatment of CNS ischemic disorders," Journal of Neurochemistry, vol. 113, no. 2, pp. 303-312, 2010.

[3] R. J. Traystman, "Animal models of focal and global cerebral ischemia," ILAR Journal, vol. 44, no. 2, pp. 85-95, 2003.

[4] J. F. Megyesi, B. Vollrath, D. A. Cook, and J. M. Findlay, "In vivo animal models of cerebral vasospasm: a review," Neurosurgery, vol. 46, no. 2, pp. 448-461, 2000.

[5] S. Ashwal and W. J. Pearce, "Animal models of neonatal stroke," Current Opinion in Pediatrics, vol. 13, no. 6, pp. 506516, 2001.

[6] M. Alonso De Leciñana, E. Díez-Tejedor, F. Carceller, and J. M. Roda, "Cerebral ischemia: from animal studies to clinical practice. Should the methods be reviewed?" Cerebrovascular Diseases, vol. 11, no. 1, supplement, pp. 20-30, 2001.

[7] X. Li, K. K. Blizzard, Z. Zeng, A. C. DeVries, P. D. Hurn, and L. D. McCullough, "Chronic behavioral testing after focal ischemia in the mouse: functional recovery and the effects of gender," Experimental Neurology, vol. 187, no. 1, pp. 94-104, 2004.

[8] K. Hattori, H. Lee, P. D. Hurn, B. J. Crain, R. J. Traystman, and A. C. DeVries, "Cognitive deficits after focal cerebral ischemia in mice," Stroke, vol. 31, no. 8, pp. 1939-1944, 2000.

[9] A. C. DeVries, R. J. Nelson, R. J. Traystman, and P. D. Hurn, "Cognitive and behavioral assessment in experimental stroke research: will it prove useful?" Neuroscience and Biobehavioral Reviews, vol. 25, no. 4, pp. 325-342, 2001.

[10] S. M. Graham, L. D. McCullough, and S. J. Murphy, "Animal models of ischemic stroke: balancing experimental aims and animal care," Comparative Medicine, vol. 54, no. 5, pp. 486496, 2004.

[11] A. Durukan and T. Tatlisumak, "Acute ischemic stroke: overview of major experimental rodent models, pathophysiology, and therapy of focal cerebral ischemia," Pharmacology Biochemistry and Behavior, vol. 87, no. 1, pp. 179-197, 2007.

[12] M. Bacigaluppi, G. Comi, and D. M. Hermann, "Animal models of ischemic stroke-part two: modeling cerebral ischemia," The Open Neurology Journal, vol. 4, pp. 34-38, 2010.

[13] M. Philip, M. Benatar, M. Fisher, and S. I. Savitz, "Methodological quality of animal studies of neuroprotective agents currently in phase II/III acute ischemic stroke trials," Stroke, vol. 40, no. 2, pp. 577-581, 2009.

[14] J. I. Rojas, M. C. Zurrú, M. Romano, L. Patrucco, and E. Cristiano, "Acute ischemic stroke and transient ischemic attack in the very old-risk factor profile and stroke subtype between patients older than 80 years and patients aged less than 80 years," European Journal of Neurology, vol. 14, no. 8, pp. 895-899, 2007.

[15] C. L. M. Sudlow and C. P. Warlow, "Comparable studies of the incidence of stroke and its pathological types: results from an international collaboration," Stroke, vol. 28, no. 3, pp. 491499, 1997.

[16] F. Liu, D. P. Schafer, and L. D. McCullough, "TTC, Fluoro-Jade $\mathrm{B}$ and NeuN staining confirm evolving phases of infarction induced by middle cerebral artery occlusion," Journal of Neuroscience Methods, vol. 179, no. 1, pp. 1-8, 2009.
[17] S. T. Carmichael, "Rodent models of focal stroke: size, mechanism, and purpose," NeuroRx, vol. 2, no. 3, pp. 396-409, 2005.

[18] C. Culmsee, C. Zhu, S. Landshamer et al., "Apoptosis-inducing factor triggered by poly(ADP-ribose) polymerase and Bid mediates neuronal cell death after oxygen-glucose deprivation and focal cerebral ischemia," Journal of Neuroscience, vol. 25, no. 44, pp. 10262-10272, 2005.

[19] L. C. Hoyte, M. Papadakis, P. A. Barber, and A. M. Buchan, "Improved regional cerebral blood flow is important for the protection seen in a mouse model of late phase ischemic preconditioning," Brain Research, vol. 1121, no. 1, pp. 231237,2006

[20] J. H. Garcia, Y. Yoshida, H. Chen et al., "Progression from ischemic injury to infarct following middle cerebral artery occlusion in the rat," American Journal of Pathology, vol. 142, no. 2, pp. 623-635, 1993.

[21] B. S. Aspey, S. Cohen, Y. Patel, M. Terruli, and M. J. G. Harrison, "Middle cerebral artery occlusion in the rat: consistent protocol for a model of stroke," Neuropathology and Applied Neurobiology, vol. 24, no. 6, pp. 487-497, 1998.

[22] M. L. Van Campagne, G. Roger Thomas, H. Thibodeaux et al., "Secondary reduction in the apparent diffusion coefficient of water, increase in cerebral blood volume, and delayed neuronal death after middle cerebral artery occlusion and early reperfusion in the rat," Journal of Cerebral Blood Flow and Metabolism, vol. 19, no. 12, pp. 1354-1364, 1999.

[23] M. Hoehn, K. Nicolay, C. Franke, and B. D. Van Sanden, "Application of magnetic resonance to animal models of cerebral Ischemia," Journal of Magnetic Resonance Imaging, vol. 14, no. 5, pp. 491-509, 2001.

[24] J. Chen, K. Jin, M. Chen et al., "Early detection of DNA strand breaks in the brain after transient focal ischemia: implications for the role of DNA damage in apoptosis and neuronal cell death," Journal of Neurochemistry, vol. 69, no. 1, pp. 232-245, 1997.

[25] M. D. Linnik, J. A. Miller, J. Sprinkle-Cavallo et al., "Apoptotic DNA fragmentation in the rat cerebral cortex induced by permanent middle cerebral artery occlusion," Molecular Brain Research, vol. 32, no. 1, pp. 116-124, 1995.

[26] P. A. Barber, L. Hoyte, D. Kirk, T. Foniok, A. Buchan, and U. Tuor, "Early T1- and T2-weighted MRI signatures of transient and permanent middle cerebral artery occlusion in a murine stroke model studied at 9.4 T," Neuroscience Letters, vol. 388, no. 1, pp. 54-59, 2005.

[27] J. Bardutzky, Q. Shen, N. Henninger, J. Bouley, T. Q. Duong, and M. Fisher, "Differences in ischemic lesion evolution in different rat strains using diffusion and perfusion imaging," Stroke, vol. 36, no. 9, pp. 2000-2005, 2005.

[28] D. Duverger and E. T. MacKenzie, "The quantification of cerebral infarction following focal ischemia in the rat: influence of strain, arterial pressure, blood glucose concentration, and age," Journal of Cerebral Blood Flow and Metabolism, vol. 8, no. 4, pp. 449-461, 1988.

[29] K. Engelhard, C. Werner, W. E. Hoffman, B. Matthes, M. Blobner, and E. Kochs, "The effect of sevoflurane and propofol on cerebral neurotransmitter concentrations during cerebral ischemia in rats," Anesthesia and Analgesia, vol. 97, no. 4, pp. 1155-1161, 2003.

[30] C. J. Cassady, J. W. Phillis, and M. H. O'Regan, "Further studies on the effects of topical lactate on amino acid efflux from the ischemic rat cortex," Brain Research, vol. 901, no. 12, pp. 30-37, 2001. 
[31] F. Liu, Z. Li, J. Li, C. Siegel, R. Yuan, and L. D. Mccullough, "Sex differences in caspase activation after stroke," Stroke, vol. 40, no. 5, pp. 1842-1848, 2009.

[32] A. Tuttolomondo, R. Di Sciacca, D. Di Raimondo, C. Renda, A. Pinto, and G. Licata, "Inflammation as a therapeutic target in acute ischemic stroke treatment," Current Topics in Medicinal Chemistry, vol. 9, no. 14, pp. 1240-1260, 2009.

[33] L. C. Schmued and K. J. Hopkins, "Fluoro-Jade B: a high affinity fluorescent marker for the localization of neuronal degeneration," Brain Research, vol. 874, no. 2, pp. 123-130, 2000.

[34] K. Tureyen, R. Vemuganti, K. A. Sailor, and R. J. Dempsey, "Infarct volume quantification in mouse focal cerebral ischemia: a comparison of triphenyltetrazolium chloride and cresyl violet staining techniques," Journal of Neuroscience Methods, vol. 139, no. 2, pp. 203-207, 2004.

[35] D. Lloyd-Jones, R. J. Adams, T. M. Brown et al., "Heart disease and stroke statistics-2010 update: a report from the American heart association," Circulation, vol. 121, no. 7, pp. e46-e215, 2010.

[36] A. S. Plump, J. D. Smith, T. Hayek et al., "Severe hypercholesterolemia and atherosclerosis in apolipoprotein E-deficient mice created by homologous recombination in ES cells," Cell, vol. 71, no. 2, pp. 343-353, 1992.

[37] T. Nabika, Z. Cui, and J. Masuda, "The stroke-prone spontaneously hypertensive rat: how good is it as a model for cerebrovascular diseases," Cellular and Molecular Neurobiology, vol. 24, no. 5, pp. 639-646, 2004.

[38] M. Watanabe, J. Osada, Y. Aratani et al., "Mice deficient in cystathionine $\beta$-synthase: animal models for mild and severe homocyst(e)inemia," Proceedings of the National Academy of Sciences of the United States of America, vol. 92, no. 5, pp. 15851589, 1995.

[39] D. Rasmussen, O. Kohler, S. Worm-Petersen et al., "Computed tomography in prognostic stroke evaluation," Stroke, vol. 23, no. 4, pp. 506-510, 1992.

[40] E. C. Anyanwu, "Neurochemical changes in the aging process: implications in medication in the elderly," TheScientific World Journal, vol. 7, pp. 1603-1610, 2007.

[41] F. Liu, R. Yuan, S. E. Benashski, and L. D. McCullough, "Changes in experimental stroke outcome across the life span," Journal of Cerebral Blood Flow and Metabolism, vol. 29, no. 4, pp. 792-802, 2009.

[42] F. Liu, P. Akella, S. E. Benashski, Y. Xu, and L. D. McCullough, "Expression of Na-K-Cl cotransporter and edema formation are age dependent after ischemic stroke," Experimental Neurology, vol. 29, no. 4, pp. 792-802, 2010.

[43] A. Jaramillo, F. Góngora-Rivera, J. Labreuche, J. J. Hauw, and P. Amarenco, "Predictors for malignant middle cerebral artery infarctions: a postmortem analysis," Neurology, vol. 66, no. 6, pp. 815-820, 2006.

[44] J. Hofmeijer, A. Algra, L. J. Kappelle, and H. B. Van Der Worp, "Predictors of life-threatening brain edema in middle cerebral artery infarction," Cerebrovascular Diseases, vol. 25, no. 1-2, pp. 176-184, 2008.

[45] P. Abete, F. Cacciatore, G. Testa et al., "Ischemic preconditioning in the aging heart: from bench to bedside," Ageing Research Reviews, vol. 9, no. 2, pp. 153-162, 2010.

[46] J. Li, Z. Zeng, B. Viollet, G. V. Ronnett, and L. D. McCullough, "Neuroprotective effects of adenosine monophosphateactivated protein kinase inhibition and gene deletion in stroke," Stroke, vol. 38, no. 11, pp. 2992-2999, 2007.
[47] L. D. McCullough, Z. Zeng, H. Li, L. E. Landree, J. McFadden, and G. V. Ronnett, "Pharmacological inhibition of AMPactivated protein kinase provides neuroprotection in stroke," Journal of Biological Chemistry, vol. 280, no. 21, pp. 2049320502, 2005.

[48] M. D. Ginsberg, "Neuroprotection for ischemic stroke: past, present and future," Neuropharmacology, vol. 55, no. 3, pp. 363-389, 2008.

[49] A. Kharlamov, E. Kharlamov, and D. M. Armstrong, "Agedependent increase in infarct volume following photochemically induced cerebral infarction: putative role of astroglia," Journals of Gerontology. Series A, vol. 55, no. 3, pp. B135-B141, 2000.

[50] G. R. Sutherland, G. A. Dix, and R. N. Auer, "Effect of age in rodent models of focal and forebrain ischemia," Stroke, vol. 27, no. 9, pp. 1663-1668, 1996.

[51] S. Shapira, M. Sapir, A. Wengier, E. Grauer, and T. Kadar, "Aging has a complex effect on a rat model of ischemic stroke," Brain Research, vol. 925, no. 2, pp. 148-158, 2002.

[52] K. P. Doyle, E. Cekanaviciute, L. E. Mamer, and M. S. Buckwalter, "TGF $\beta$ signaling in the brain increases with aging and signals to astrocytes and innate immune cells in the weeks after stroke," Journal of Neuroinflammation, vol. 7, article no. 62,2010

[53] R. Y. Wang, P. S. G. Wang, and Y. R. Yang, "Effect of age in rats following middle cerebral artery occlusion," Gerontology, vol. 49, no. 1, pp. 27-32, 2003.

[54] D. Poli, E. Antonucci, E. Grifoni, R. Abbate, G. F. Gensini, and D. Prisco, "Gender differences in stroke risk of atrial fibrillation patients on oral anticoagulant treatment," Thrombosis and Haemostasis, vol. 101, no. 5, pp. 938-942, 2009.

[55] D. M. Kent, A. M. Buchan, and M. D. Hill, "The gender effect in stroke thrombolysis: of CASES, controls, and treatmenteffect modification," Neurology, vol. 71, no. 14, pp. 1080-1083, 2008.

[56] E. D. Hall and R. J. Traystman, "Role of animal studies in the design of clinical trials," Frontiers of Neurology and Neuroscience, vol. 25, pp. 10-33, 2009.

[57] G. Lanzino, N. F. Kassell, N. W. C. Dorsch et al., "Doubleblind, randomized, vehicle-controlled study of high-dose tirilazad mesylate in women with aneurysmal subarachnoid hemorrhage - part I. A cooperative study in Europe, Australia, New Zealand, and South Africa," Journal of Neurosurgery, vol. 90, no. 6, pp. 1011-1017, 1999.

[58] R. L. Sacco et al., "American Heart Association Prevention Conference. IV. Prevention and rehabilitation of stroke. Risk factors," Stroke, vol. 28, no. 7, pp. 1507-1517, 1997.

[59] N. K. Wenger, L. Speroff, and B. Packard, "Cardiovascular health and disease in women," New England Journal of Medicine, vol. 329, no. 4, pp. 247-256, 1993.

[60] A. Paganini-Hill, "Hormone replacement therapy and stroke: risk, protection or no effect?" Maturitas, vol. 38, no. 3, pp. 243-261, 2001.

[61] J. W. Simpkins, S. H. Yang, Y. Wen, and M. Singh, "Estrogens, progestins, menopause and neurodegeneration: basic and clinical studies," Cellular and Molecular Life Sciences, vol. 62, no. 3, pp. 271-280, 2005.

[62] L. D. McCullough, N. J. Alkayed, R. J. Traystman, M. J. Williams, and P. D. Hurn, "Postischemic estrogen reduces hypoperfusion and secondary ischemia after experimental stroke," Stroke, vol. 32, no. 3, pp. 796-802, 2001.

[63] L. D. McCullough and P. D. Hurn, "Estrogen and ischemic neuroprotection: an integrated view," Trends in Endocrinology and Metabolism, vol. 14, no. 5, pp. 228-235, 2003. 
[64] N. J. Alkayed, S. J. Murphy, R. J. Traystman, and P. D. Hurn, "Neuroprotective effects of female gonadal steroids in reproductively senescent female rats," Stroke, vol. 31, no. 1, pp. 161-168, 2000.

[65] T. J. Toung, T. Y. Chen, M. T. Littleton-Kearney, P. D. Hurn, and S. J. Murphy, "Effects of combined estrogen and progesterone on brain infarction in reproductively senescent female rats," Journal of Cerebral Blood Flow and Metabolism, vol. 24, no. 10, pp. 1160-1166, 2004.

[66] S. J. Murphy, L. D. McCullough, and J. M. Smith, "Stroke in the female: role of biological sex and estrogen," ILAR journal / National Research Council, Institute of Laboratory Animal Resources, vol. 45, no. 2, pp. 147-159, 2004.

[67] R. A. Lobo, "Menopause and stroke and the effects of hormonal therapy," Climacteric, vol. 10, no. 2, supplement, pp. 27-31, 2007.

[68] S. Wassertheil-Smoller, S. L. Hendrix, M. Limacher et al., "Effect of estrogen plus progestin on stroke in postmenopausal women-the women's health initiative: a randomized trial," Journal of the American Medical Association, vol. 289, no. 20, pp. 2673-2684, 2003.

[69] S. Suzuki, C. M. Brown, C. D. Dela Cruz, E. Yang, D. A. Bridwell, and P. M. Wise, "Timing of estrogen therapy after ovariectomy dictates the efficacy of its neuroprotective and antiinflammatory actions," Proceedings of the National Academy of Sciences of the United States of America, vol. 104, no. 14, pp. 6013-6018, 2007.

[70] R. L. Prentice, R. Langer, M. L. Stefanick et al., "Combined postmenopausal hormone therapy and cardiovascular disease: toward resolving the discrepancy between observational studies and the Women's Health Initiative clinical trial," American Journal of Epidemiology, vol. 162, no. 5, pp. 404-414, 2005.

[71] R. L. Prentice, R. D. Langer, M. L. Stefanick et al., "Combined analysis of women's health initiative observational and clinical trial data on postmenopausal hormone treatment and cardiovascular disease," American Journal of Epidemiology, vol. 163, no. 7, pp. 589-599, 2006.

[72] J. O. Strom, E. Theodorsson, L. Holm, and A. Theodorsson, "Different methods for administering $17 \beta$-estradiol to ovariectomized rats result in opposite effects on ischemic brain damage," BMC Neuroscience, vol. 11, article no. 39, 2010.

[73] T. D. Farr, H. V. O. Carswell, L. Gallagher et al., "17 $\beta$-Estradiol treatment following permanent focal ischemia does not influence recovery of sensorimotor function," Neurobiology of Disease, vol. 23, no. 3, pp. 552-562, 2006.

[74] J. T. Lang and L. D. McCullough, "Pathways to ischemic neuronal cell death: are sex differences relevant?" Journal of Translational Medicine, vol. 6, article no. 33, 2008.

[75] S. Renolleau, S. Fau, C. Goyenvalle et al., "Specific caspase inhibitor Q-VD-OPh prevents neonatal stroke in P7 rat: a role for gender," Journal of Neurochemistry, vol. 100, no. 4, pp. 1062-1071, 2007.

[76] L. D. McCullough, Z. Zeng, K. K. Blizzard, I. Debchoudhury, and P. D. Hurn, "Ischemic nitric oxide and poly (ADP-ribose) polymerase-1 in cerebral ischemia: male toxicity, female protection," Journal of Cerebral Blood Flow and Metabolism, vol. 25, no. 4, pp. 502-512, 2005.

[77] M. Yuan, C. Siegel, Z. Zeng, J. Li, F. Liu, and L. D. McCullough, "Sex differences in the response to activation of the poly (ADP-ribose) polymerase pathway after experimental stroke," Experimental Neurology, vol. 217, no. 1, pp. 210-218, 2009.

[78] J. Li and L. D. McCullough, "Sex differences in minocyclineinduced neuroprotection after experimental stroke," Journal of
Cerebral Blood Flow and Metabolism, vol. 29, no. 4, pp. 670674, 2009.

[79] J. L. LaPrairie and A. Z. Murphy, "Female rats are more vulnerable to the long-term consequences of neonatal inflammatory injury," Pain, vol. 132, no. 1, pp. S124-S133, 2007.

[80] G. Candore, C. R. Balistreri, F. Listì et al., "Immunogenetics, gender, and longevity," Annals of the New York Academy of Sciences, vol. 1089, pp. 516-537, 2006.

[81] M. De la Fuente, I. Baeza, N. Guayerbas et al., "Changes with ageing in several leukocyte functions of male and female rats," Biogerontology, vol. 5, no. 6, pp. 389-400, 2004.

[82] J. K. Relton, D. Martin, R. C. Thompson, and D. A. Russell, "Peripheral administration of interleukin-1 receptor antagonist inhibits brain damage after focal cerebral ischemia in the rat," Experimental Neurology, vol. 138, no. 2, pp. 206-213, 1996.

[83] E. Tarkowski, L. Rosengren, C. Blomstrand et al., "Early intrathecal production of interleukin- 6 predicts the size of brain lesion in stroke," Stroke, vol. 26, no. 8, pp. 1393-1398, 1995.

[84] C. Siegel, C. Turtzo, and L. D. McCullough, "Sex differences in cerebral ischemia: possible molecular mechanisms," Journal of Neuroscience Research, vol. 88, no. 13, pp. 2765-2774, 2010. 


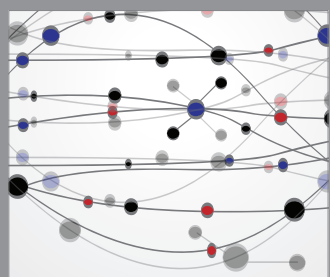

The Scientific World Journal
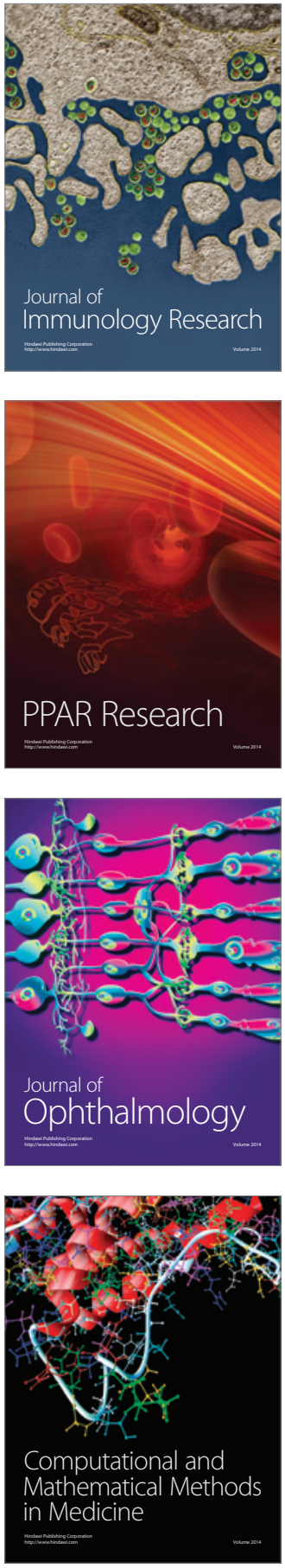

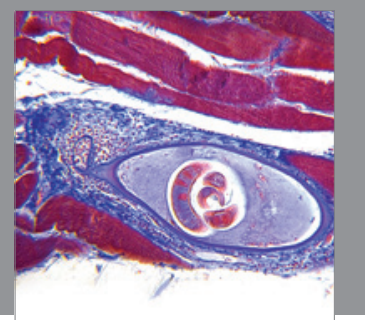

Gastroenterology

Research and Practice
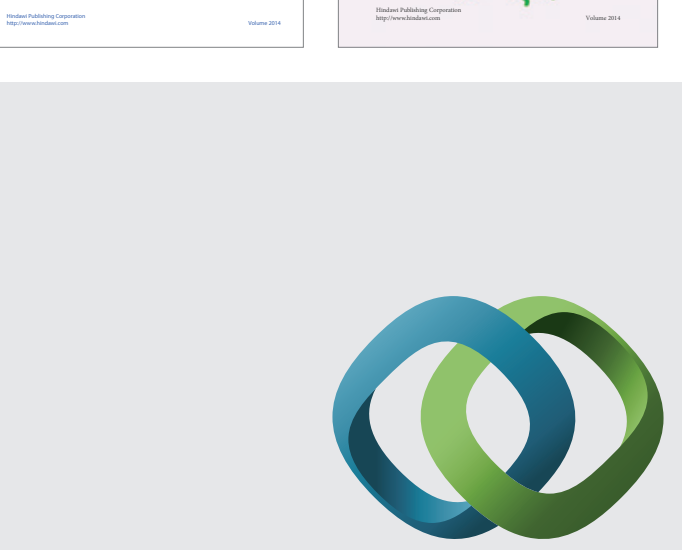

\section{Hindawi}

Submit your manuscripts at

http://www.hindawi.com
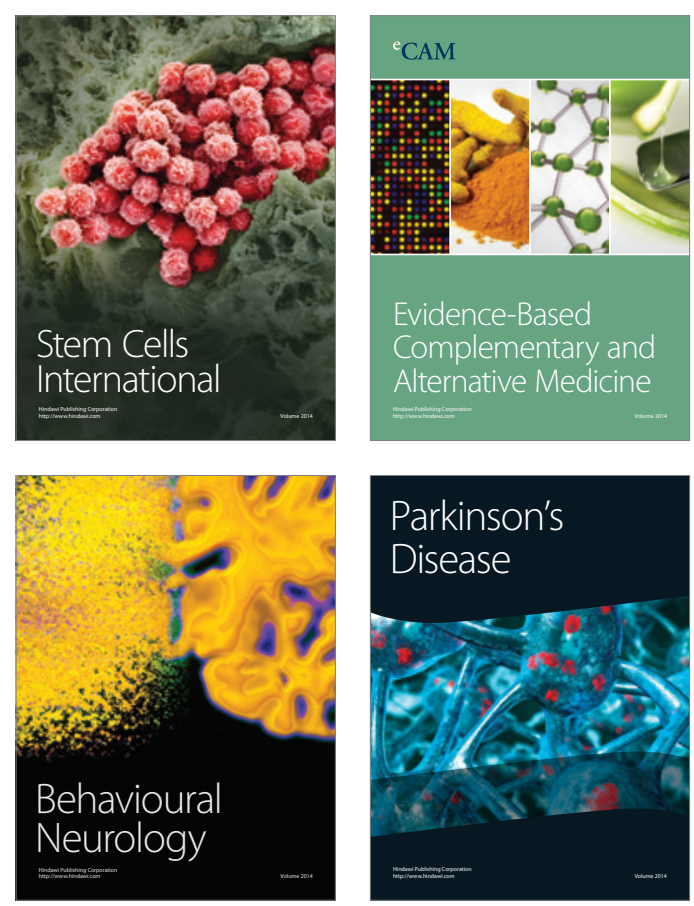

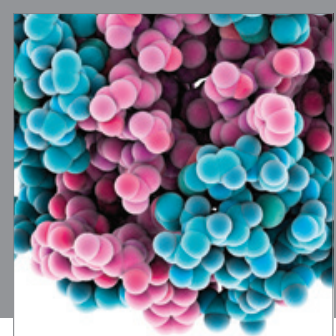

Journal of
Diabetes Research

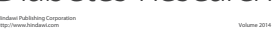

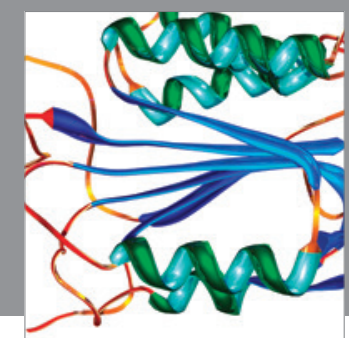

Disease Markers
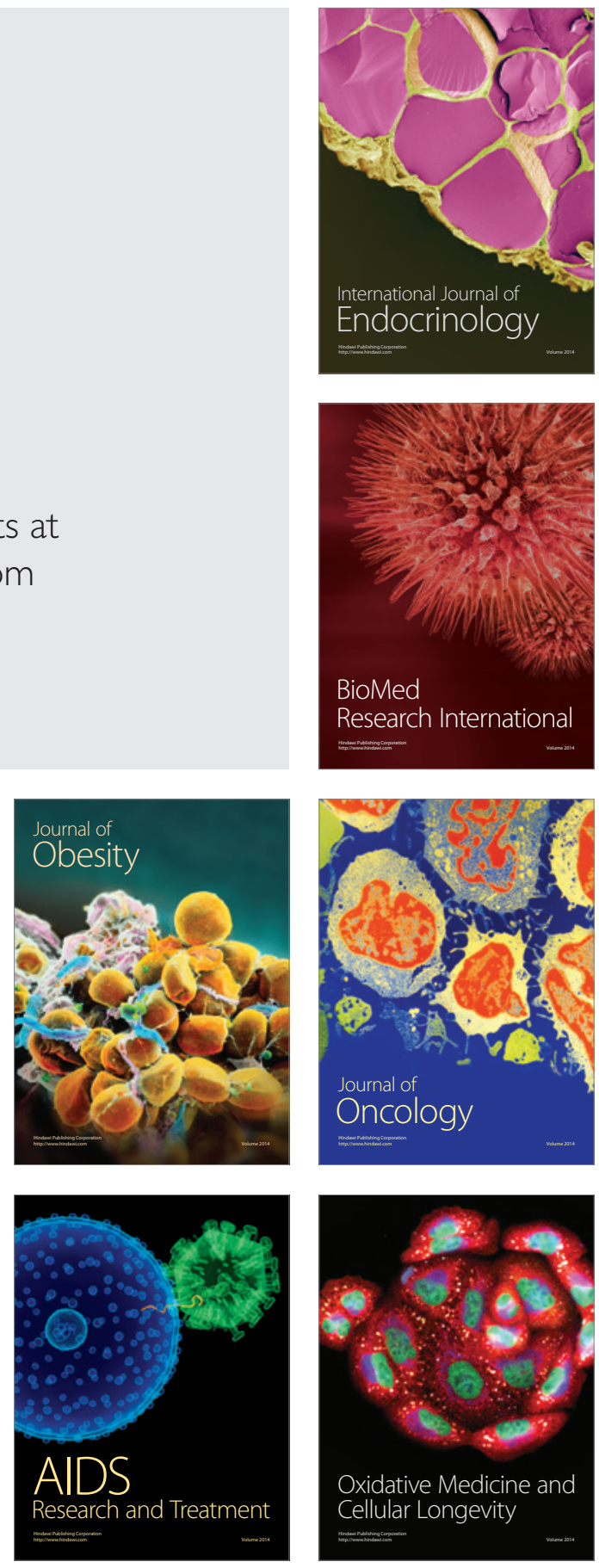